TRANSACTIONS OF THE

AMERICAN MATHEMATICAL SOCIETY

Volume 349, Number 9, September 1997, Pages 3787-3802

S $0002-9947(97) 01861-8$

\title{
LINE BUNDLE LAPLACIANS OVER ISOSPECTRAL NILMANIFOLDS
}

\author{
DOROTHEE SCHUETH
}

\begin{abstract}
We show that nontrivial isospectral deformations of a big class of compact Riemannian two-step nilmanifolds can be distinguished from trivial deformations by the behaviour of bundle Laplacians on certain non-flat hermitian line bundles over these manifolds.
\end{abstract}

\section{INTRODUCTION}

Let $N$ be a simply-connected nilpotent Lie group, $\Gamma$ a cocompact discrete subgroup of $N$, and $g$ a left invariant metric on $N$ which thus descends to a metric, again denoted by $g$, on $\Gamma \backslash N$. Let $\Phi_{t}$ be a continuous family of so-called $\Gamma$-almost inner automorphisms (see Definiton 1.2). Then a theorem of C. Gordon and E. Wilson ([GW], 1984) implies that $\left(\Gamma \backslash N, \Phi_{t}^{*} g\right)$ is an isospectral deformation; i.e., the metrics in this continuous family all have the same spectrum for the Laplacian acting on functions. Moreover, such a deformation is nontrivial (i.e., the manifolds involved are not pairwise isometric) except in the case where the $\Phi_{t}$ differ from each other by inner automorphisms. By results of D. DeTurck and C. Gordon ([DG1], [DG2]), the $\left(\Gamma \backslash N, \Phi_{t}^{*} g\right)$ are even strongly isospectral; i.e., they are isospectral also for every other natural elliptic operator, for example for the Laplacian acting on $p$-forms.

On the other hand, H. Ouyang and H. Pesce $[\mathrm{OP}]$ proved that in the case where the Lie group $N$ is two-step nilpotent, every continuous family of metrics $g_{t}$ on $\Gamma \backslash N$ which are induced from left invariant metrics on $N$ and which are pairwise isospectral for the Laplacian on functions must arise in the above way. In particular, two-step nilpotency of $N$ implies that every continuous family of isospectral left invariant metrics $g_{t}$ on $\Gamma \backslash N$ is also strongly isospectral. Hence it is not possible to distinguish the nontrivial ones among these deformations from the trivial ones by studying the spectrum of other natural elliptic operators over the underlying Riemannian manifolds.

Although it is often possible to detect the changing geometry in isospectral deformations by direct geometric approaches (see for example [DGGW1], [DGGW2], $[\mathrm{Sch}]$ ), it is interesting to see if one can distinguish the manifolds by spectral properties as well. P. Gilkey raised the question of whether for the above isospectral deformations $\left(\Gamma \backslash N, \Phi_{t}^{*} g\right)$ this can be done by studying the Laplacian acting on other flat vector bundles over these manifolds; he had shown in [Gi] that Ikeda's

Received by the editors April 15, 1996.

1991 Mathematics Subject Classification. Primary 58G25; Secondary 22E30, 22E25.

Key words and phrases. Line bundles, Laplacian, isospectral deformations, nilmanifolds.

Supported by a grant from DFG, Bonn/Germany; partially supported by SFB 256, Bonn. 
examples of isospectral lens spaces [Ik] can be distinguished by the spectra of certain operators with coefficients in flat bundles. But it turns out (see [GOS]) that the above deformations are isospectral also for the Laplacian on every flat vector bundle over the underlying manifolds.

This is the motivation for considering non-flat hermitian line bundles $E$ over the base manifold $\Gamma \backslash N$. If $\left(\Gamma \backslash N, \Phi_{t}^{*} g\right)$ is an isospectral deformation as above and $\nabla_{t}$ is a family of metric connections on $E$, consider the bundle Laplacian associated with $\Phi_{t}^{*} g$ and $\nabla_{t}$. Its spectrum can in general be either constant or nonconstant in $t$, depending on the choice of the family $\nabla_{t}$; He Ouyang studied several explicit examples in the two-step nilpotent case in $[\mathrm{Ou}]$.

But suppose one can find a hermitian line bundle $E$ over $\Gamma \backslash N$ such that no choice of metric connections $\nabla_{t}$ leads to isospectral bundle Laplacians on $E$. Then this will indeed spectrally detect the nontriviality of the underlying deformation $\left(\Gamma \backslash N, \Phi_{t}^{*} g\right)$, as desired.

The question of whether for every nontrivial isospectral deformation $\left(\Gamma \backslash N, \Phi_{t}^{*} g\right)$ of a two-step nilmanifold $\Gamma \backslash N$ there exists a hermitian line bundle $E$ over $\Gamma \backslash N$ with the above property was raised by C. Gordon (in a slightly more special version) and remained open in Ouyang's work [Ou].

The goal of this paper is to give an affirmative answer (Theorem 1.15) to this question under a certain generic condition on $\Gamma \backslash N$ and under certain restrictions on the choice of the family $\nabla_{t}$ (namely, the $\nabla_{t}$ are required to be invariant in the sense of Definition 1.13(i) and to depend continuously on $t$ in the sense of Definition 1.13(ii)). These restrictions on the family $\nabla_{t}$ do not change the fact that the existence of a bundle $E$ as in our theorem is a property which spectrally distinguishes nontrivial underlying deformations from trivial ones. The additional condition assumed for $\Gamma \backslash N$ is that $N$ should not admit any 4-fold $\Gamma$-almost inner automorphisms (see Definition 1.2(ii)) which are not inner. We conjecture that this condition is actually unnecessary.

In the proof of the theorem, as well as in the definition of invariant connections in 1.13 we use the fact that the $S^{1}$-principal bundle associated with $E$ is always isomorphic to a bundle of the form $\pi: \tilde{\Gamma} \backslash \tilde{N} \rightarrow \Gamma \backslash N$, where $\tilde{N}$ is a one-dimensional central extension of $N$, and $\tilde{\Gamma}$ is a cocompact discrete subgroup of $\tilde{N}$ such that $\pi(\tilde{\Gamma})=\Gamma$.

The paper is organized as follows:

In $\S 1$, we begin by giving the necessary background on isospectral deformations via almost inner automorphisms, the bundle Laplacian on hermitian line bundles, and principal $S^{1}$-bundles over compact nilmanifolds. Our main result (Theorem 1.15) is then first stated in an a priori weaker formulation (Theorem 1.7). After making the necessary definitions we give the more intrinsic formulation 1.15, which we finally show to be equivalent to 1.7 .

In $\S 2$ we construct, for any given two-step nilpotent Lie group $N$ and a cocompact discrete subgroup $\Gamma$ of $N$, certain one-dimensional central extensions $\tilde{N}$ and $\tilde{\Gamma}$ as above such that $\tilde{N}$ is again two-step nilpotent and such that no automorphism of $N$ which is not 4 -fold $\Gamma$-almost inner can be extended to a $\tilde{\Gamma}$-almost inner automorphism of $\tilde{N}$ (Theorem 2.2).

In $\S 3$ we then show that - after eventually refining $\tilde{\Gamma}$ - the hermitian line bundle $E$ associated with the $S^{1}$-principal bundle $\tilde{\Gamma} \backslash \tilde{N}$ satisfies indeed the statement of the theorem. Note that the bundle Laplacian on $E$ is - up to the addition of some 
constant multiple of the identity - unitarily equivalent to the Laplacian acting on a certain subspace of functions on $\tilde{\Gamma} \backslash \tilde{N}$, where $\tilde{\Gamma} \backslash \tilde{N}$ is endowed with a certain Riemannian metric associated with the metric on the base manifold and the bundle connection. Since $\tilde{\Gamma} \backslash \tilde{N}$ was constructed in $\S 2$ to be two-step nilpotent, we can use H. Pesce's $[\mathrm{Pe}]$ calculations concerning the spectrum of two-step nilmanifolds in order to achieve the goal of $\S 3$.

The author would like to thank Carolyn Gordon for drawing her attention to this problem and for several helpful discussions. Moreover she would like to thank Dartmouth College for its hospitality.

\section{Preliminaries and formulation of the Main Theorem}

\section{Isospectral deformations of compact nilmanifolds.}

Let $(M, g)$ be a compact Riemannian manifold, and let $\Delta_{g}$ be the Laplacian acting on functions by

$$
\left(\Delta_{g} f\right)(p):=-\left.\sum_{i=1}^{n} \frac{d^{2}}{d t^{2}}\right|_{t=0} f\left(c_{i}(t)\right) \quad \text { for } p \in M,
$$

where the $c_{i}$ are geodesics starting in $p$ such that $\left\{\dot{c}_{1}(0), \ldots, \dot{c}_{n}(0)\right\}$ is an orthonormal basis for $T_{p} M$. The discrete sequence $0=\lambda_{0}<\lambda_{1} \leq \lambda_{2} \leq \ldots \rightarrow \infty$ of the eigenvalues of $\Delta_{g}$, counted with the corresponding multiplicities, is called the spectrum of $(M, g)$. Two compact Riemannian manifolds are said to be isospectral if their spectra are equal.

In the following, let $N$ be a simply-connected nilpotent Lie group with Lie algebra $\mathcal{N}$. Note that in this case, the group exponential map exp $: \mathcal{N} \rightarrow N$ is a diffeomorphism. Denote its inverse by log.

1.1 Remark. If $\mathcal{N}$ admits a basis $\mathcal{B}$ with respect to which the structure coefficients are rational, then the $\operatorname{set} \exp (\mathcal{B}) \subset N$ generates a cocompact discrete subgroup $\Gamma$ of $N$. Conversely, if $N$ admits a cocompact discrete subgroup $\Gamma$, then $\operatorname{span}_{\mathbf{z}}(\log \Gamma)$ is a lattice of full rank in $\mathcal{N}$, each of whose elements is a rational multiple of an element of $\log \Gamma$; the structure coefficients with respect to any basis of this lattice are rational. (See $[\mathrm{Ra}]$ for these facts.) We call $X \in \mathcal{N}$ a $\Gamma$-rational vector if $X \in \operatorname{span}_{\mathbf{Q}}(\log \Gamma)$, and a linear subspace of $\mathcal{N}$ is called $\Gamma$-rational if it is spanned by $\Gamma$-rational vectors, or equivalently, if it is spanned by elements of $\log \Gamma$.

In the following, let $\Gamma$ be a cocompact discrete subgroup of $N$.

1.2 Definition. (i) The group $\operatorname{AIA}(N ; \Gamma)$ of $\Gamma$-almost inner automorphisms of $N$ and the Lie algebra $\operatorname{AID}(\mathcal{N} ; \Gamma)$ of $\Gamma$-almost inner derivations of $\mathcal{N}$ are defined as follows:

$$
\begin{aligned}
& \operatorname{AIA}(N ; \Gamma):=\left\{\Phi \in \operatorname{Aut}(N) \mid \forall \gamma \in \Gamma \exists a \in N: \Phi(\gamma)=a \gamma a^{-1}\right\}, \\
& \operatorname{AID}(\mathcal{N} ; \Gamma):=\{\varphi \in \operatorname{Der}(\mathcal{N}) \mid \forall X \in \log \Gamma \exists A \in \mathcal{N}: \varphi(X)=[A, X]\} .
\end{aligned}
$$

(ii) An automorphism $\Phi$ of $N$, resp. derivation $\varphi$ of $\mathcal{N}$, is called $p$-fold ( $\Gamma$-) almost inner if for every $p$-dimensional ( $\Gamma$-rational) subspace $\mathcal{U} \in \mathcal{N}$ there exists $a \in N$ such that $\left.\Phi_{* e}\right|_{\mathcal{U}}=\left.\operatorname{Ad}_{a}\right|_{\mathcal{U}}$, resp. there exists $A \in \mathcal{N}$ such that $\left.\varphi\right|_{\mathcal{U}}=\left.\operatorname{ad}_{A}\right|_{\mathcal{U}}$.

According to [Go], $\operatorname{AIA}(N ; \Gamma)$ is a Lie subgroup of $\operatorname{Aut}(N)$, contained in the subgroup of unipotent automorphisms, and its Lie algebra is $\operatorname{AID}(\mathcal{N} ; \Gamma)$; more precisely, $\operatorname{AID}(\mathcal{N} ; \Gamma)=\left\{\log \Phi_{* e} \mid \Phi \in \operatorname{AIA}(N ; \Gamma)\right\}$. It is easy to see that the same 
holds for the $p$-fold $\Gamma$-almost inner automorphisms and derivations. The group $\operatorname{Inn}(N)$ of inner automorphisms is clearly contained in $\operatorname{AIA}(N ; \Gamma)$; in general, this inclusion is nontrivial. Note that $\operatorname{AIA}(N ; \Gamma)$ just consists of the 1 -fold $\Gamma$-almost inner automorphisms.

Every left invariant metric $g$ on $N$ descends to a metric on the compact manifold $\Gamma \backslash N$. By abuse of notation we will call this metric left invariant again, and we will denote it by the same name as its pullback to $N$. For $\Phi \in \operatorname{Aut}(N)$ the metric $\Phi^{*} g$ is again left invariant and thus descends to the compact manifold $\Gamma \backslash N$.

1.3 Theorem ([GW], [DG2], [Go]). If $\Phi \in \operatorname{AIA}(N ; \Gamma)$ and $g$ is left invariant, then the Riemannian nilmanifolds $(\Gamma \backslash N, g)$ and $\left(\Gamma \backslash N, \Phi^{*} g\right)$ are isospectral.

In particular, if $\Phi_{t}$ is a continuous family of automorphisms in $\operatorname{AIA}(N ; \Gamma)$ with $\Phi_{0}=\mathrm{Id}$, then $\left(\Gamma \backslash N, \Phi_{t}^{*} g\right)$ is an isospectral deformation. This deformation is called trivial if the manifolds involved are pairwise isometric. As shown in [GW], $\left(\Gamma \backslash N, \Phi_{t}^{*} g\right)$ is a trivial deformation if and only if $\Phi_{t} \in \operatorname{Inn}(N)$ for every $t$.

Now assume that $N$ is two-step nilpotent; i.e., we have $[\mathcal{N}, \mathcal{N}] \subseteq \mathcal{Z}$, where $\mathcal{Z}$ denotes the center of $\mathcal{N}$. In this case it is known that the above method is the only way of constructing isospectral deformations $\left(\Gamma \backslash N, g_{t}\right)$ with left invariant metrics $g_{t}$ :

1.4 Theorem ([Pe], $[\mathrm{Ou}],[\mathrm{OP}])$. Let $N$ be two-step nilpotent, $\Gamma$ a cocompact discrete subgroup of $N$, and $g_{t}$ a continuous family of left invariant metrics such that $\left(\Gamma \backslash N, g_{t}\right)$ is an isospectral deformation. Then there exists a continuous family $\Phi_{t} \in \operatorname{AIA}(N ; \Gamma)$ with $\Phi_{0}=\operatorname{Id}$ and $g_{t}=\Phi_{t}^{*} g_{0}$.

\section{The bundle Laplacian on hermitian line bundles.}

Let $M$ be a smooth manifold, and let $\pi: E \rightarrow M$ be a complex line bundle over $M$. Assume that $E$ is endowed with a smooth hermitian fiber metric $\langle.,$.$\rangle .$ Let $P:=\{u \in E \mid\langle u, u\rangle=1\} \subset E$. Obviously $P$ can be considered as a principal $S^{1}$-bundle with which $E$ is associated via the canonical representation $S^{1} \rightarrow U(1)$.

Denote the space of smooth sections of $E$ by $\mathcal{E}(E)$. There is a canonical bijection $\alpha: \mathcal{E}(E) \rightarrow C(P)^{S^{1}}$, where

$$
C(P)^{S^{1}}:=\left\{f \in C^{\infty}(P, \mathbf{C}) \mid f(u z)=z^{-1} \cdot f(u) \forall u \in P \forall z \in S^{1}\right\} .
$$

For $\Psi \in \mathcal{E}(E)$ the function $f=\alpha(\Psi) \in C(P)^{S^{1}}$ is given by the condition $\Psi(\pi(u))=$ $u \cdot f(u)$ for all $u \in P$.

There is a canonical correspondence between connections in $P$ (that is, $S^{1}$-invariant distributions on $P$ which are complementary to the fiber direction) and metric connections on $E$. In the following, consider a given connection $\mathcal{H}$ in $P$ and the corresponding metric connection $\nabla$ on $E$. Denote the space of smooth vector fields on $M$ by $V(M)$, and denote the unique $\mathcal{H}$-horizontal lift of $X \in V(M)$ to $P$ by $X^{*}$. Then we have (see $\left.[\mathrm{KN}]\right)$

$$
\alpha\left(\nabla_{X}\left(\alpha^{-1}(f)\right)=X^{*}(f) \text { for all } X \in V(M) \text { and } f \in C(P)^{S^{1}} .\right.
$$

In the following we assume that $M$ is compact and $g$ is a Riemannian metric on $M$. The bundle Laplacian $\nabla^{*} \nabla: \mathcal{E}(E) \rightarrow \mathcal{E}(E)$ is given by

$$
\left(\nabla^{*} \nabla \Psi\right)(p)=-\sum_{i=1}^{n}\left(\nabla_{e_{i}(p)} \nabla_{e_{i}} \Psi-\nabla_{\tilde{\nabla}_{e_{i}(p)} e_{i}} \Psi\right),
$$

where $\widetilde{\nabla}$ is the Levi-Cività connection associated with the metric $g$ on $M$, and $e_{1}, \ldots, e_{n}$ are vector fields in some neighbourhood of $p$ such that $\left\{e_{1}(p), \ldots, e_{n}(p)\right\}$ 
is an orthonormal basis of $T_{p} M$. It is well-known that $\nabla^{*} \nabla$ is an elliptic selfadjoint positive semi-definite operator with discrete spectrum consisting of real nonnegative eigenvalues tending to infinity. Each eigenvalue occurs with finite multiplicity, and every eigenvector is a smooth section of $E$. Denote the spectrum of $\nabla^{*} \nabla$ by $\operatorname{spec}(g, \nabla)$. Equation (2) implies that under conjugation with $\alpha$ the bundle Laplacian corresponds to the operator $\tilde{\Delta}: C(P)^{S^{1}} \rightarrow C(P)^{S^{1}}$ defined by

$$
(\tilde{\Delta} f)(u)=-\sum_{i=1}^{n}\left(\left.e_{i}^{*}\right|_{u}\left(e_{i}^{*}(f)\right)-\left.\left(\widetilde{\nabla}_{e_{i}} e_{i}\right)^{*}\right|_{u}(f)\right),
$$

where $e_{1}, \ldots, e_{n}$ are vector fields as in the definition of $\nabla^{*} \nabla($ with $p:=\pi(u))$.

1.5 Remark. Consider the unique Riemannian metric $\tilde{g}$ on $P$ such that $\pi:(P, \tilde{g}) \rightarrow$ $(M, g)$ is a Riemannian submersion, the fibers of $P$ are $\tilde{g}$-vertical to the $\mathcal{H}$-horizontal subspaces, $\tilde{g}$ is $S^{1}$-invariant, and the fibers of $P$ have length 1 . Let $W$ be the $S^{1}$ invariant unit vector field tangent to the fibers of $P$; i.e., for $u \in P$ we have $W(u)=\left.\frac{d}{d s}\right|_{s=0} e^{2 \pi i s} u$. Denote the Laplacian of the Riemannian manifold $(P, \tilde{g})$ by $\Delta_{\tilde{g}}$. Then obviously $\tilde{\Delta}(f)=\Delta_{\tilde{g}}(f)+W^{2}(f)=\Delta_{\tilde{g}}(f)-4 \pi^{2} f$ for all $f \in C(P)^{S^{1}}$; thus

$$
\tilde{\Delta}=\left.\left(\Delta_{\tilde{g}}-4 \pi^{2} \mathrm{Id}\right)\right|_{C(P)^{S^{1}}} .
$$

It is easily seen that if we consider the $L^{2}$-scalar products induced on $\mathcal{E}(E)$ by $g$, resp. induced on $C(P)^{S^{1}}$ by $\tilde{g}$, then $\alpha$ is an isometry between these two spaces. Thus $\nabla^{*} \nabla$ is unitarily equivalent to $\left.\left(\Delta_{\tilde{g}}-4 \pi^{2} \mathrm{Id}\right)\right|_{C(P)^{S^{1}}}$. Denote the spectrum of this last operator by $\operatorname{spec}(g, \mathcal{H})$; then in particular, $\operatorname{spec}(g, \mathcal{H})=\operatorname{spec}(g, \nabla)$.

\section{Principal $S^{1}$-bundles over compact nilmanifolds.}

In the following we assume $M=\Gamma \backslash N$, where $N$ is a simply-connected nilpotent Lie group and $\Gamma$ is a cocompact discrete subgroup of $N$.

1.6 Remark. Let $P$ be a principal $S^{1}$-bundle over $\Gamma \backslash N$. It is well-known that $P$ is isomorphic to a bundle of the form $\pi: \tilde{\Gamma} \backslash \tilde{N} \rightarrow \Gamma \backslash N$, where $\tilde{N}$ is a simply-connected one-dimensional central extension of $N$, the corresponding projection is $\pi: \tilde{N} \rightarrow N$, and $\tilde{\Gamma}$ is a discrete cocompact subgroup of $\tilde{N}$ with $\pi(\tilde{\Gamma})=\Gamma$. The Lie group $\tilde{N}$ is nilpotent again, and $\tilde{N}$ and $\tilde{\Gamma}$ are determined uniquely up to isomorphism. In the following we will call vector fields and forms on compact nilmanifolds left invariant if the corresponding objects on the universal cover are left invariant. The same holds for connections in $\tilde{\Gamma} \backslash \tilde{N}$, viewed as distributions.

Now we can give the first formulation of the result:

1.7 Main Theorem (first formulation). Let $N$ be a simply-connected two-step nilpotent Lie group, and let $\Gamma$ be a cocompact discrete subgroup of $N$. Suppose that $N$ does not admit any 4-fold $\Gamma$-almost inner automorphisms which are not inner. Let $g_{t}(t \geq 0)$ be a continuous family of isospectral left invariant metrics on $\Gamma \backslash N$. If the deformation $\left(\Gamma \backslash N, g_{t}\right)$ is nontrivial then there exist a one-dimensional central extension $\tilde{N}$ of $N$ and a cocompact discrete subgroup $\tilde{\Gamma}$ of $\tilde{N}$ whose projection to $N$ is $\Gamma$, such that for every continuous family of left invariant connections $\mathcal{H}_{t}$ in the $S^{1}$-bundle $\pi: \tilde{\Gamma} \backslash \tilde{N} \rightarrow \Gamma \backslash N$, the spectrum $\operatorname{spec}\left(g_{t}, \mathcal{H}_{t}\right)$ is nonconstant in $t$.

1.8 Remark. In view of equation (3) above, this theorem can be restated as follows: Under the assumptions of 1.7 , there exist a one-dimensional central extension $\tilde{N}$ 
of $N$ and a cocompact discrete subgroup $\tilde{\Gamma}$ of $\tilde{N}$ whose projection to $N$ is $\Gamma$, such that the following holds:

(*) If $\tilde{g}_{t}$ is any continuous family of left invariant metrics on $\tilde{\Gamma} \backslash \tilde{N}$ with the property that for every $t$, $\pi:\left(\tilde{\Gamma} \backslash \tilde{N}, \tilde{g}_{t}\right) \rightarrow\left(\Gamma \backslash N, g_{t}\right)$ is a Riemannian submersion, and

(+) $\quad$ the $\tilde{g}_{t}$-length of the fibers of $\tilde{\Gamma} \backslash \tilde{N}$, viewed as an $S^{1}$-bundle over $\Gamma \backslash N$, is equal to one,

then the spectrum of $\left.\Delta_{\tilde{g}_{t}}\right|_{C(\tilde{\Gamma} \backslash \tilde{N})^{S^{1}}}$ is nonconstant in $t$.

1.9 Observations. (i) In the situation of $1.7 / 1.8$, we know by 1.4 that $g_{t}$ is of the form $\Phi_{t}^{*} g_{0}$ for some continuous family $\Phi_{t} \in \operatorname{AIA}(N ; \Gamma)$. Now suppose that $\tilde{N}$ and $\tilde{\Gamma}$ are such that the family $\Phi_{t}$ extends to a continuous family in $\operatorname{AIA}(\tilde{N} ; \tilde{\Gamma})$; i.e., there exists a continuous family $\tilde{\Phi}_{t} \in \operatorname{AIA}(\tilde{N} ; \tilde{\Gamma})$ such that $\pi \circ \tilde{\Phi}_{t}=\Phi_{t} \circ \pi$ and $\tilde{\Phi}_{t *}(W)=$ $W$ for $W$ as in Remark 1.5. Then $\tilde{\Gamma} \backslash \tilde{N}$ can certainly not have property $(*)$. In fact, if $\tilde{g}$ is any left invariant metric on $\tilde{\Gamma} \backslash \tilde{N}$ satisfying condition $(+)$, then $\tilde{g}_{t}:=\tilde{\Phi}_{t}^{*} \tilde{g}$ will also satisfy $(+)$ for every $t$. But then by 1.3 , the spectrum of $\Delta_{\tilde{g}_{t}}$ will be constant in $t$, and by continuity of the eigenvalues this will also hold for the restriction $\left.\Delta_{\tilde{g}_{t}}\right|_{C(\tilde{\Gamma} \backslash \tilde{N})^{S^{1}}}$.

(ii) In particular, $\tilde{\Gamma} \backslash \tilde{N}$ cannot satisfy (*) if it admits a flat left invariant connection. In this case, the horizontal distribution is integrable and thus $\tilde{N}$ is isomorphic to $N \times \mathbf{R}$. But then $\tilde{\Phi}_{t}:=\left(\Phi_{t}\right.$, Id $)$ is obviously a $\tilde{\Gamma}$-almost inner extension of $\Phi_{t}$ to $\tilde{N}$.

This leads to the following

\subsection{Strategy for proving Theorem 1.7:}

(a) Construct a one-dimensional central extension $\tilde{N}$ of $N$ and a cocompact discrete subgroup $\tilde{\Gamma}$ of $\tilde{N}$ such that $\tilde{N}$ is still two-step nilpotent, and such that no automorphism of $N$ which is not 4 -fold $\Gamma$-almost inner can extend to a $\tilde{\Gamma}$-almost inner automorphism of $\tilde{N}$. This will be the topic of $\S 2$ below.

(b) If $\tilde{\Gamma} \backslash \tilde{N}$ has been constructed as in (a), it follows from 1.4 that for any continuous family $\tilde{g}_{t}$ of left invariant metrics on $\tilde{\Gamma} \backslash \tilde{N}$ which satisfy $(+)$, the spectrum of $\Delta_{\tilde{g}_{t}}$ is nonconstant in $t$. In order to make sure that this is the case already for the restriction $\left.\Delta_{\tilde{g}_{t}}\right|_{C(\tilde{\Gamma} \backslash \tilde{N})^{S^{1}}}$, one has to eventually refine $\tilde{\Gamma}$ and to do some careful analysis of the spectrum of $\Delta_{\tilde{g}_{t}}$, following Pesce's work [Pe] on the spectrum of two-step nilmanifolds. This will be done in $\S 3$ of this paper.

1.11 Remarks. (i) We conjecture that the assumption that $N$ does not admit any 4 -fold $\Gamma$-almost inner, non-inner automorphisms can be removed. At the end of $\S 2$ it will become clear, however, what difficulties arise - at least in our approach if we do not make this assumption.

(ii) Note that the assumption just mentioned is not very restrictive, because "generically" a non-inner, almost inner automorphism is only 1 -fold almost inner. The latter ones are not at all scarce. For example, every two-step nilpotent Lie group $N$ which is nonsingular in the sense of [Eb] and whose center is not one-dimensional admits plenty of non-inner, almost inner automorphisms. But higher-fold almost inner, non-inner automorphisms are much harder to find. See [DGGW2] for specific examples of $p$-fold almost inner, non-inner automorphisms for arbitrary $p$. 


\section{Intrinsic reformulation of the result.}

Before carrying out the above strategy in the subsequent sections, we give a second formulation of the Main Theorem (see 1.15 below) in which the statement is formulated in terms of a hermitian line bundle $E$ over $\Gamma \backslash N$ and some intrinsically defined space $\mathcal{L}(E)$ of "invariant" metric connections on $E$. Note that in the previous formulation 1.7, the set of left invariant connections on $\tilde{\Gamma} \backslash \tilde{N}$ is not intrinsically defined in terms of just the bundle structure of $\tilde{\Gamma} \backslash \tilde{N}$, viewed as an $S^{1}$-bundle over $\Gamma \backslash N$. The reason is that there are bundle isomorphisms from $\tilde{\Gamma} \backslash \tilde{N}$ to itself which induce the identity on $\Gamma \backslash N$ but do not preserve the nilmanifold structure of $\tilde{\Gamma} \backslash \tilde{N}$; in particular, they do not preserve the set of left invariant connections on $\tilde{\Gamma} \backslash \tilde{N}$. We need the following preparations and definitions:

1.12 Notation and Remarks. Let $P$ be a principal $S^{1}$-bundle over a Riemannian manifold $(M, g)$.

(i) A bundle isomorphism $F: P \rightarrow P$ is called a gauge transformation if $F$ induces the identity on $M$. If $\mathcal{H}$ is a connection in $P$, and if $\tilde{g}$ and $\tilde{g}^{\prime}$ are the Riemannian metrics on $P$ corresponding to the connections $\mathcal{H}$ and $F_{*} \mathcal{H}$ as in Remark 1.5, then $\tilde{g}=F^{*} \tilde{g}^{\prime}$; in particular, $\Delta_{\tilde{g}^{\prime}}=F^{*-1} \circ \Delta_{\tilde{g}} \circ F^{*}$. The space $C(P)^{S^{1}}$ is invariant under $F^{*}$; hence the bundle Laplacians belonging to $\mathcal{H}$ and $F_{*} \mathcal{H}$ are unitarily equivalent, and $\operatorname{spec}(g, \mathcal{H})=\operatorname{spec}\left(g, F_{*} \mathcal{H}\right)$. By the same argument, if $P^{\prime}$ is another $S^{1}$-bundle over $M$, and if $j: P \rightarrow P^{\prime}$ is a bundle isomorphism inducing the identity on $M$, then $\operatorname{spec}(g, \mathcal{H})=\operatorname{spec}\left(g, j_{*} \mathcal{H}\right)$ for every connection $\mathcal{H}$ in $P$.

(ii) A 1-form $\vartheta$ on $P$ is called a connection form if $\vartheta$ is $S^{1}$-invariant and $\vartheta(W)=$ 1 for the vertical vector field $W$ from Remark 1.5. Note that connections and connection forms on $P$ are in one-to-one correspondence; $\vartheta$ is the connection form corresponding to $\mathcal{H}$ if $\operatorname{ker} \vartheta=\mathcal{H}$. In this context, we will also write $\operatorname{spec}(g, \vartheta)$ instead of $\operatorname{spec}(g, \mathcal{H})$. If $F$ is a gauge transformation then $F^{*-1} \vartheta$ corresponds to $F_{*} \mathcal{H}$.

1.13 Definition. Let $P$ be a principal $S^{1}$-bundle over a compact nilmanifold $\Gamma \backslash N$, and let $\tilde{N}$ and $\tilde{\Gamma}$ be as in Remark 1.6.

(i) Let $\mathcal{L}(P):=\left\{j^{*} \vartheta \mid j: P \rightarrow \tilde{\Gamma} \backslash \tilde{N}\right.$ is a bundle isomorphism inducing the identity on $\Gamma \backslash N$, and $\vartheta$ is a left invariant connection form on $\tilde{\Gamma} \backslash \tilde{N}\}$, and $\mathcal{L}(E):=$ $\{\nabla \mid \nabla$ is a metric connection on $E$ corresponding to some $\vartheta \in \mathcal{L}(P)\}$ for the associated hermitian line bundle $E$. We call elements of $\mathcal{L}(P)$ or $\mathcal{L}(E)$ invariant connections.

(ii) Fix an arbitrary Riemannian metric $h$ on $P$. On the space of 1-forms $\lambda$ on $P$ consider the $L^{1}$-norm $\|\lambda\|:=\int_{P}|\lambda(u)| d v o l_{h}(u)$, where $|$.$| is the norm induced by h$ on $T_{u}^{*} P$. We call a family of 1 -forms $\lambda_{t}$ on $P$ continuous if it is continuous with respect to $\|$.$\| . Similarly, we call a family of metric connections on E$ continuous if it corresponds to a continuous family of connection forms on $P$.

1.14 Remarks. (i) Note that the set $\mathcal{L}(P)$ does not depend on the specific choice of $\tilde{N}$ and $\tilde{\Gamma}$, since these are unique up to isomorphism (see Remark 1.6), and isomorphisms between Lie groups preserve left invariance of differential forms.

(ii) If $\vartheta \in \mathcal{L}(P)$ then also $F^{*} \vartheta \in \mathcal{L}(P)$ for every gauge transformation $F$. But two invariant connection forms $\vartheta_{1}, \vartheta_{2}$ on $P$ need not necessarily differ by a gauge transformation, since two left invariant connection forms on $\tilde{\Gamma} \backslash \tilde{N}$ do not need to do so. 
(iii) Note that the notion of continuity in 1.13(ii) is independent of the choice of $h$. In fact, a different choice would give rise to an equivalent norm $\|$. $\|$ because $P$ is compact.

With the above definitions we have:

1.15 Main Theorem (second formulation). Under the assumptions of 1.7, there exists a hermitian line bundle $E$ over $\Gamma \backslash N$ such that for every continuous family of invariant metric connections $\nabla_{t} \in \mathcal{L}(E)$ the spectrum $\operatorname{spec}\left(g_{t}, \nabla_{t}\right)$ of the corresponding bundle Laplacians is nonconstant in $t$.

By part (ii) of the following lemma, this second formulation is indeed equivalent to 1.7 :

1.16 Lemma. Let $P$ be a principal $S^{1}$-bundle over a compact nilmanifold $\Gamma \backslash N$, and let $j: P \rightarrow \tilde{\Gamma} \backslash \tilde{N}$ be a bundle isomorphism which induces the identity on $\Gamma \backslash N$, where $\tilde{N}$ and $\tilde{\Gamma}$ are as in Remark 1.6. Let $g$ be an arbitrary Riemannian metric on $\Gamma \backslash N$.

(i) $\left(j^{-1}\right)^{*} \mathcal{L}(P)=\{\eta+\beta \mid \eta$ is a left invariant connection form on $\tilde{\Gamma} \backslash \tilde{N}$, and $\beta$ is exact and vanishes in the fiber direction $\}$.

(ii) Let $\vartheta_{t} \in \mathcal{L}(P)$ be a continuous family of invariant connection forms on $P$. Then there exists a family $\eta_{t}$ of left invariant connection forms on $\tilde{\Gamma} \backslash \tilde{N}$ with the following properties: $\operatorname{spec}\left(g, \eta_{t}\right)=\operatorname{spec}\left(g, \vartheta_{t}\right)$ for every $t$, and the $\eta_{t}$ depend continuously on $t$ in the elementary sense when viewed as elements of the dual space $\tilde{\mathcal{N}}^{*}$, where $\tilde{\mathcal{N}}$ denotes the Lie algebra of $\tilde{N}$.

Proof. (i) " $\subseteq$ ": Let $\vartheta \in\left(j^{-1}\right)^{*} \mathcal{L}(P)$. By definition of $\mathcal{L}(P)$ we have $\vartheta=F^{*} \vartheta_{1}$ for some gauge transformation $F$ of $\tilde{\Gamma} \backslash \tilde{N}$ and some left invariant connection form $\vartheta_{1}$. Since $d \vartheta_{1}$ is horizontal and $F$ induces the identity on $\Gamma \backslash N$, it follows that $d \vartheta=$ $F^{*} d \vartheta_{1}=d \vartheta_{1}$. In particular, $d \vartheta$ is left invariant. By Nomizu's theorem [No], the inclusion of the space of left invariant forms into the space of all differential forms on a compact nilmanifold induces an isomorphism on the cohomology level (with respect to the Lie algebra cohomology with real coefficients, resp. the DeRham cohomology of the manifold). Thus $\vartheta=\eta^{\prime}+\nu$, where $\eta^{\prime}$ is left invariant and $\nu$ is closed. Again by Nomizu's theorem, $\nu=\eta^{\prime \prime}+\beta$, where $\eta^{\prime \prime}$ is left invariant and closed, and $\beta$ is exact. The statement follows by letting $\eta:=\eta^{\prime}+\eta^{\prime \prime}$; note that $\beta$, being exact and $S^{1}$-invariant, must vanish in the fiber direction.

" $\supseteq$ ": Let $\eta$ be a left invariant connection form and $\beta$ an exact form on $\tilde{\Gamma} \backslash \tilde{N}$ which vanishes in the fiber direction. Choose a function $f$ on $\tilde{\Gamma} \backslash \tilde{N}$ such that $\beta=d f$. Then $f$ is constant on the fibers; let $F$ be the gauge transformation of the $S^{1}$-bundle $\tilde{\Gamma} \backslash \tilde{N}$ defined by $F(u)=e^{2 \pi i f(u)} u$ for $u \in \tilde{\Gamma} \backslash \tilde{N}$. Then $F^{*} \eta=\eta+d f=\eta+\beta$. Since $F \circ j: P \rightarrow \tilde{\Gamma} \backslash \tilde{N}$ still induces the identity on $\Gamma \backslash N$, we have $(F \circ j)^{*} \eta \in \mathcal{L}(P)$; thus $\eta+\beta=\left(j^{-1}\right)^{*}(F \circ j)^{*} \eta$ lies in $\left(j^{-1}\right)^{*} \mathcal{L}(P)$.

(ii) For every $t$ we know by (i) that $\left(j^{-1}\right)^{*} \vartheta_{t}$ is of the form $\eta_{t}+\beta_{t}$, where $\eta_{t}$ is left invariant and $\beta_{t}$ is exact. As in the proof of (i) we conclude that $\left(j^{-1}\right)^{*} \vartheta_{t}=F_{t}^{*} \eta_{t}$ for some gauge transformation $F_{t}$, and thus $\operatorname{spec}\left(g, \eta_{t}\right)=\operatorname{spec}\left(g, \vartheta_{t}\right)$. It remains to show that the $\eta_{t} \in \tilde{\mathcal{N}}^{*}$ are continuous in $t$. Let $m$ be a left invariant metric on $\tilde{\Gamma} \backslash \tilde{N}$ and $X$ a left invariant vector field. Then $\int_{\tilde{\Gamma} \backslash \tilde{N}} \beta_{t}(X) d v o l_{m}=0$ since $\beta_{t}$ is exact. Hence we have $\int_{\tilde{\Gamma} \backslash \tilde{N}}\left(\left(j^{-1}\right)^{*} \vartheta_{t}\right)(X) d v \operatorname{vol}_{m}=\int_{\tilde{\Gamma} \backslash \tilde{N}} \eta_{t}(X) d \operatorname{vol}_{m}=\operatorname{vol}_{m}(\tilde{\Gamma} \backslash \tilde{N}) \cdot \eta_{t}(X)$. Let $h:=j^{*} m$. Then $\left|\int_{\tilde{\Gamma} \backslash \tilde{N}}\left(\left(j^{-1}\right)^{*}\left(\vartheta_{s}-\vartheta_{t}\right)\right)(X) d v o l_{m}\right|=\left|\int_{P}\left(\vartheta_{s}-\vartheta_{t}\right)\left(j_{*}^{-1} X\right) d v o l_{h}\right|$ 
$\leq|X|_{m} \cdot\left\|\vartheta_{s}-\vartheta_{t}\right\| \rightarrow 0$, for $s \rightarrow t$ since $\vartheta_{t}$ was assumed to be continuous in the sense of Definition 1.13. By the previous equation, this implies $\eta_{s}(X) \rightarrow \eta_{t}(X)$ for $s \rightarrow t$. Since $X \in \tilde{\mathcal{N}}$ was arbitrary, the statement follows.

\section{Extendability of ALMOST INNER AUTOMORPhisms}

In this section we carry out step (a) of Strategy 1.10. The central result of this section is Theorem 2.2 .

2.1 Notation and Remarks. Let $N$ be a simply-connected nilpotent Lie group with Lie algebra $\mathcal{N}$, and let $\Gamma$ be a cocompact discrete subgroup of $N$.

(i) Let $\omega \in \mathcal{N}^{*} \wedge \mathcal{N}^{*}$ be a closed 2-form on $\mathcal{N}$; i.e., $\omega(X,[Y, V])+\omega(Y,[V, X])+$ $\omega(V,[X, Y])=0$ for all $X, Y, V \in \mathcal{N}$. Associated with $\omega$ there is a one-dimensional central extension $\mathcal{N}_{\omega}$ of $\mathcal{N}$ which is defined as follows: Introduce a Lie algebra structure on the vector space $\mathcal{N} \times \mathbf{R}$ by letting

$$
[(X, r),(Y, s)]_{\omega}=([X, Y], \omega(X, Y))
$$

for $X, Y \in \mathcal{N}$ and $r, s \in \mathbf{R}$. The fact that $\omega$ is closed is equivalent to the Jacobi identity for $[., .]_{\omega}$. Denote $\mathcal{N} \times \mathbf{R}$, equipped with this Lie algebra structure, by $\mathcal{N}_{\omega}$. We identify $\mathcal{N}$ with the corresponding subspace of $\mathcal{N}_{\omega}$ and denote the new central vector $(0,1) \in \mathcal{N}_{\omega}$ by $W$. Thus we write elements of $\mathcal{N}_{\omega}$ in the form $X+r W$ with $X \in \mathcal{N}$ and $r \in \mathbf{R}$.

(ii) We call a form $\omega$ as above horizontal if $\omega(\mathcal{N},[\mathcal{N}, \mathcal{N}])=\{0\}$. Note that a horizontal form is automatically closed. If $\mathcal{N}$ is $p$-step nilpotent $(p \geq 2)$ and $\omega$ is horizontal, then $\mathcal{N}_{\omega}$, too, is $p$-step nilpotent.

(iii) Denote by $N_{\omega}$ the simply-connected Lie group with Lie algebra $\mathcal{N}_{\omega}$, and denote the exponential map from $\mathcal{N}_{\omega}$ to $N_{\omega}$ by $\exp _{\omega}$. If $N$ is two-step nilpotent and $\omega$ is horizontal, then the Campbell-Baker-Hausdorff formula implies that multiplication in $N_{\omega}$ is given by

$$
\begin{aligned}
& \exp _{\omega}(X+r W) \cdot \exp _{\omega}(Y+s W) \\
& \quad=\exp _{\omega}\left(\log (\exp X \cdot \exp Y)+\left(r+s+\frac{1}{2} \omega(X, Y)\right) W\right)
\end{aligned}
$$

for $X, Y \in \mathcal{N}$ and $r, s \in \mathbf{R}$, where exp denotes the exponential map from $\mathcal{N}$ to $N$ and $\log$ its inverse.

(iv) Suppose $N$ is two-step nilpotent and $\omega$ is a horizontal 2-form on $\mathcal{N}$ with the property $\omega(\log \Gamma, \log \Gamma) \subseteq 2 \mathbf{Z}$. Then

$$
\Gamma_{\omega}:=\exp _{\omega}(\log \Gamma+\mathbf{Z} W)
$$

is a subgroup of $N_{\omega}$ by (iii). Obviously $\Gamma_{\omega}$ is discrete and cocompact, and its projection to $N$ is $\Gamma$. Note also that if $X \in \mathcal{N}$ is $\Gamma$-rational, then the corresponding vector $X \in \mathcal{N}_{\omega}$ (as in (i)) is $\Gamma_{\omega}$-rational.

(v) Let $\Phi \in \operatorname{Aut}(N)$. Then $\tilde{\Phi} \in \operatorname{Aut}\left(N_{\omega}\right)$ is called an extension of $\Phi$ if $\pi \circ \tilde{\Phi}=\Phi \circ \pi$ and $\tilde{\Phi}_{*}(W)=W$. Similarly, if $\varphi \in \operatorname{Der}(\mathcal{N})$ then $\tilde{\varphi} \in \operatorname{Der}\left(\mathcal{N}_{\omega}\right)$ is called an extension of $\varphi$ if $\pi \circ \tilde{\varphi}=\varphi \circ \pi$ and $\tilde{\varphi}(W)=0$. Here, $\pi: N_{\omega} \rightarrow N$, resp. $\pi: \mathcal{N}_{\omega} \rightarrow \mathcal{N}$, denote the canonical projections. Note that if $\Phi_{* e}=\exp \varphi$ then $\tilde{\varphi} \in \operatorname{Der}\left(\mathcal{N}_{\omega}\right)$ is an extension of $\varphi$ if and only if $\tilde{\Phi}$ with $\tilde{\Phi}_{* e}=\tilde{\varphi}$ is an extension of $\Phi$.

(vi) If $\varphi$ is a $(\Gamma$-)almost inner derivation of $\mathcal{N}$ then $\varphi(X) \subseteq[\mathcal{N}, \mathcal{N}]$ for all $(\Gamma$ rational) vectors $X \in \mathcal{N}$; thus $\varphi(\mathcal{N}) \subseteq[\mathcal{N}, \mathcal{N}]$. Similarly, $\varphi(\mathcal{Z})=\{0\}$, where $\mathcal{Z}$ denotes the center of $\mathcal{N}$; note that $\mathcal{Z}$ is a $\Gamma$-rational subspace of $\mathcal{N}$ (see [Ra]). In particular, if $\mathcal{N}$ is two-step nilpotent then $\varphi^{2}=0$, and thus $\exp \varphi=\operatorname{Id}+\varphi$. 
2.2 Theorem. Let $N$ be a two-step nilpotent Lie group with Lie algebra $\mathcal{N}$, and let $\Gamma$ be a cocompact discrete subgroup of $N$. Let $\Phi$ be an automorphism of $N$ which is not 4-fold $\Gamma$-almost inner. Then there exists a horizontal 2-form $\omega$ on $\mathcal{N}$ with $\omega(\log \Gamma, \log \Gamma) \subseteq 2 \mathbf{Z}$ such that $\Phi$ does not extend to a $\Gamma_{\omega}$-almost inner automorphism on the associated central extension $N_{\omega}$.

Proof. If $\Phi$ is not even 1-fold $\Gamma$-almost inner then obviously the trivial central extension associated with $\omega=0$ satisfies the statement. Thus suppose $\Phi \in \operatorname{AIA}(N ; \Gamma)$.

For simplicity, we call $\Gamma$-rational vectors and subspaces of $\mathcal{N}$ just "rational" in the following; linear maps will be called rational if they send rational vectors to rational vectors. Let $\varphi:=\log \Phi_{* e}=\Phi_{* e}-\operatorname{Id} \in \operatorname{AID}(\mathcal{N} ; \Gamma)$, and let $\mathcal{V}$ be a rational complement of $\mathcal{Z}$ in $\mathcal{N}$. Let $\mathcal{U} \in \mathcal{N}$ be a rational subspace on which $\varphi$ differs from every inner derivation $\operatorname{ad}_{X}(X \in \mathcal{N})$. Since $\left.\varphi\right|_{\mathcal{Z}}=0$ by $2.1(\mathrm{vi})$, we can assume $\mathcal{U} \subseteq \mathcal{V}$. Let $\left\{X_{1}, \ldots, X_{p}\right\}$ be a rational basis of $\mathcal{U}$. By our hypothesis that $\Phi$ is not 4 -fold $\Gamma$-almost inner, we know that we can choose $\mathcal{U}$ such that $p \leq 4$; however, for the time being, we let $p$ be general in order to illustrate later the problem that arises if we drop this hypothesis and only assume that $\Phi$ is not inner.

For every pair $i, j \in\{1, \ldots, p\}$ define a linear map $b_{i j} \in \operatorname{Hom}(\mathcal{U}, \mathcal{Z})$ by $b_{i j}\left(X_{i}\right)=$ $-\left[X_{i}, X_{j}\right], b_{i j}\left(X_{j}\right)=\left[X_{i}, X_{j}\right], b_{i j}\left(X_{k}\right)=0$ for $k \neq i, j$. Let $B=\operatorname{span}\left\{b_{i j} \mid i, j \in\right.$ $\{1, \ldots, p\}\}$.

Choose a rational basis $\left\{Z_{1}, \ldots, Z_{m}\right\}$ of $\mathcal{Z}$. Introduce a scalar product $\langle.,$. on $\mathcal{U}$, respectively on $\mathcal{Z}$, such that $\left\{X_{1}, \ldots, X_{p}\right\}$, respectively $\left\{Z_{1}, \ldots, Z_{m}\right\}$, is an orthonormal basis. Consider the associated scalar product on $\operatorname{Hom}(\mathcal{U}, \mathcal{Z})$. Note that $\left.\varphi\right|_{\mathcal{U}} \in \operatorname{Hom}(\mathcal{U}, \mathcal{Z})$ by $2.1($ vi). In the following, we will always write $\varphi$ for $\left.\varphi\right|_{\mathcal{U}}, \operatorname{ad}_{X}$ for $\left.\operatorname{ad}_{X}\right|_{\mathcal{U}}, \operatorname{ad}_{\mathcal{N}}$ for $\left.\operatorname{ad}_{\mathcal{N}}\right|_{\mathcal{U}}$. Note that $\operatorname{ad}_{\mathcal{N}}$ and $B$ are rational subspaces of $\operatorname{Hom}(\mathcal{U}, \mathcal{Z})$. We know that $\varphi \notin \operatorname{ad}_{\mathcal{N}}$.

Case 1. Suppose we even have

$$
\varphi \notin \operatorname{ad}_{\mathcal{N}}+B .
$$

Then choose a rational linear map $f \in \operatorname{Hom}(\mathcal{U}, \mathcal{Z})$ such that

$$
f \perp \operatorname{ad}_{\mathcal{N}}, \quad f \perp B, \quad f \not \perp \varphi .
$$

Define a horizontal 2-form $\omega$ on $\mathcal{N}$ by letting

$$
\begin{gathered}
\left.\omega\right|_{\mathcal{N} \times \mathcal{U}}\left(Y, X_{j}\right)=\left\langle\left[Y, X_{j}\right], f\left(X_{j}\right)\right\rangle \quad \text { for } j=1, \ldots, p, \\
\left.\omega\right|_{\mathcal{U} \times \mathcal{N}}=-\left.\omega\right|_{\mathcal{N} \times \mathcal{U}},\left.\quad \omega\right|_{\overline{\mathcal{U}} \times \overline{\mathcal{U}}}=0,
\end{gathered}
$$

where $\overline{\mathcal{U}}$ is some rational complement of $\mathcal{U}$ in $\mathcal{N}$ which contains $\mathcal{Z}$. Note that $f \perp B$ is equivalent to $\omega \mid \mathcal{U} \times \mathcal{U}$ indeed being skew-symmetric. In fact, for $i, j \in\{1, \ldots, p\}$ we have $\omega\left(X_{i}, X_{j}\right)+\omega\left(X_{j}, X_{i}\right)=\left\langle b_{i j}\left(X_{j}\right), f\left(X_{j}\right)\right\rangle+\left\langle b_{i j}\left(X_{i}\right), f\left(X_{i}\right)\right\rangle=\left\langle f, b_{i j}\right\rangle=0$. Moreover, note that $\omega$ takes rational values on pairs of rational vectors by the choice of $f$. By eventually multiplying $f$ by some non-zero scalar factor, we can assume $\omega(\log \Gamma, \log \Gamma) \subseteq 2 \mathbf{Z}$.

We claim that $\varphi$ does not extend to a $\Gamma_{\omega}$-almost inner derivation of $\mathcal{N}_{\omega}$ (where $\Gamma_{\omega}$ is as in 2.1(vi)), and more precisely: Every extension $\tilde{\varphi}$ of $\varphi$ fails to be almost inner on at least one of the $\Gamma_{\omega}$-rational vectors $X_{1}, \ldots, X_{p}, X_{1}+\cdots+X_{p} \in \mathcal{N} \subset \mathcal{N}_{\omega}$. In fact, if there is $Y_{j} \in \mathcal{N} \subset \mathcal{N}_{\omega}$ such that, in the notation 2.1(i), $\tilde{\varphi}\left(X_{j}\right)=\left[Y_{j}, X_{j}\right]_{\omega}=$ $\left[Y_{j}, X_{j}\right]+\omega\left(Y_{j}, X_{j}\right) W$, then $\left[Y_{j}, X_{j}\right]=\varphi\left(X_{j}\right)$ since $\tilde{\varphi}$ extends $\varphi$. Hence

$$
\tilde{\varphi}\left(X_{j}\right)=\varphi\left(X_{j}\right)+\left\langle\varphi\left(X_{j}\right), f\left(X_{j}\right)\right\rangle W \quad \text { for } j=1, \ldots, p .
$$


In particular, $\tilde{\varphi}\left(X_{1}+\cdots+X_{p}\right)$ has $W$-component $\langle\varphi, f\rangle \neq 0$. On the other hand, for every $Y \in \mathcal{N} \subset \mathcal{N}_{\omega}$, the $W$-component of $\left[Y, X_{1}+\cdots+X_{p}\right]$ is

$$
\omega\left(Y, X_{1}+\cdots+X_{p}\right)=\sum_{j=1}^{n}\left\langle\left[Y, X_{j}\right], f\left(X_{j}\right)\right\rangle=\left\langle\operatorname{ad}_{Y}, f\right\rangle=0 .
$$

Hence $\tilde{\varphi}$ cannot be almost inner on $X_{1}+\cdots+X_{p}$ if it is almost inner on each of the vectors $X_{1}, \ldots, X_{p}$. Now, if $\tilde{\Phi}$ were any $\Gamma_{\omega}$-almost inner extension of $\Phi$ to $N_{\omega}$, then $\tilde{\varphi}:=\log \tilde{\Phi}_{* e}$ would be a $\Gamma_{\omega}$-almost inner extension of $\varphi$ to $\mathcal{N}_{\omega}$, in contradiction to what we just showed. Thus the statement is proved for the case $\varphi \notin \operatorname{ad}_{\mathcal{N}}+B$.

Case 2. Now suppose $\varphi \in \operatorname{ad}_{\mathcal{N}}+B$. Note that the space $B$ depends on the basis $\left\{X_{1}, \ldots, X_{p}\right\}$ of $\mathcal{U}$. Our strategy now is to construct a new rational basis $\left\{\tilde{X}_{1}, \ldots, \tilde{X}_{p}\right\}$ of $\mathcal{U}$ such that for the corresponding space

$$
\begin{gathered}
\tilde{B}:=\operatorname{span}\left\{\tilde{b}_{i j} \mid i, j \in\{1, \ldots, p\}\right\}, \text { where } \\
\tilde{b}_{i j}\left(\tilde{X}_{i}\right)=-\left[\tilde{X}_{i}, \tilde{X}_{j}\right], \tilde{b}_{i j}\left(\tilde{X}_{j}\right)=\left[\tilde{X}_{i}, \tilde{X}_{j}\right], \tilde{b}_{i j}\left(\tilde{X}_{k}\right)=0 \text { for } k \neq i, j,
\end{gathered}
$$

we will have $\varphi \notin \operatorname{ad}_{\mathcal{N}}+\tilde{B}$. Then we will be in the situation of Case 1 again, for which the statement is already proved.

Since adding an inner derivation to $\varphi$ affects neither its non-innerness nor its extendability properties, we can assume without loss of generality that $\varphi \in B$; in particular,

$$
\varphi(\mathcal{U}) \subseteq[\mathcal{U}, \mathcal{U}]
$$

Consider the space $H:=\operatorname{Hom}(\mathcal{U}, \mathcal{U} \wedge \mathcal{U})$ of linear maps from $\mathcal{U}$ to $\mathcal{U} \wedge \mathcal{U}$. Define $\ell: \mathcal{U} \wedge \mathcal{U} \rightarrow \mathcal{Z}$ by $\ell: X \wedge Y \mapsto[X, Y]$, and let $L=(\ell \circ):. H \rightarrow \operatorname{Hom}(\mathcal{U}, \mathcal{Z})$. Let $A=\operatorname{span}\left\{A_{X} \mid X \in \mathcal{U}\right\}$, where $A_{X}(Y)=X \wedge Y$ for $X, Y \in \mathcal{U}$. Note that $L(A)=\operatorname{ad}_{\mathcal{U}}$. Choose $\psi \in H$ such that $L(\psi)=\varphi$ (this is possible by (5)). Let $\mathcal{T}=\left\{X \in \mathcal{N} \mid \operatorname{ad}_{X}(\mathcal{U}) \subseteq[\mathcal{U}, \mathcal{U}]\right\}$, and define $A^{\prime}=\left\{f \in H \mid L(f) \in \operatorname{ad}_{\mathcal{T}}\right\}$. Let $K=\operatorname{ker} L \subseteq H$. Note that $A^{\prime}$ and $K$ are rational subspaces of $H$, and $A \subseteq A^{\prime}$. We know that $\psi \notin A^{\prime}+K$, because otherwise, by applying $L$, we would get $\varphi \in \operatorname{ad}_{\mathcal{T}}$, in contradiction to the non-innerness of $\varphi$. On $\mathcal{U} \wedge \mathcal{U}$ and $H$ consider the canonical scalar products associated with the above scalar product on $\mathcal{U}$. Choose a rational map $f_{0} \in H$ such that

$$
f_{0} \perp A^{\prime}, \quad f_{0} \perp K, \quad f_{0} \not \perp \psi .
$$

By $f_{0} \perp A \subseteq A^{\prime}$ we have

$$
\sum_{j=1}^{p}\left\langle f_{0}\left(X_{j}\right), X \wedge X_{j}\right\rangle=0 \quad \text { for all } X \in \mathcal{U} .
$$

Viewing $f_{0}$ as an element of $\mathcal{U}^{*} \otimes \mathcal{U} \wedge \mathcal{U} \cong H$, this just translates to the condition

$$
\iota\left(f_{0}\right)=0,
$$

where $\iota: \mathcal{U}^{*} \otimes \mathcal{U} \wedge \mathcal{U} \rightarrow \mathcal{U}$ is the canonical contraction defined by $\iota: \lambda \otimes(X \wedge Y) \mapsto$ $\lambda(X) Y-\lambda(Y) X$. Let

$$
S=\left\{f \in H \mid\left\langle f\left(X_{i}\right), X_{i} \wedge X_{j}\right\rangle=\left\langle f\left(X_{j}\right), X_{i} \wedge X_{j}\right\rangle \text { for all } i, j \in\{1, \ldots, p\}\right\} .
$$

Every $g \in G l(\mathcal{U})$ acts canonically on $H$ by $g(f): X \mapsto g_{*} f g^{-1}(X)$, where $g_{*}(Y \wedge V)=g Y \wedge g V$. If Conjecture 2.3 below is true in dimension $p$, then (7) 
implies that there exists a rational $g \in G l(\mathcal{U})$ such that

$$
g\left(f_{0}\right) \in S \text {. }
$$

Let $h={ }^{t} g: \mathcal{U} \rightarrow \mathcal{U}$ be the transposed map with respect to the above scalar product. Define $\tilde{X}_{j}=h\left(X_{j}\right)$ for $j=1, \ldots, p$, and let $\tilde{\perp}$ denote orthogonality with respect to the scalar product on $H$ associated with the rational basis $\left\{\tilde{X}_{1}, \ldots, \tilde{X}_{p}\right\}$ of $\mathcal{U}$. By (8), we have $f_{0} \perp h\left(S^{\perp}\right)=(h S)^{\widetilde{\perp}}$. Together with (6), this implies

$$
\psi \notin A^{\prime}+(h S)^{\tilde{\perp}}+K .
$$

Note that for the space $\tilde{B} \subseteq \operatorname{Hom}(\mathcal{U}, \mathcal{Z})$ associated with the basis $\left\{\tilde{X}_{1}, \ldots, \tilde{X}_{p}\right\}$ as in (4), we have $L\left((h S)^{\tilde{\perp}}\right)=\tilde{B}$. Thus, applying $L$ to $(9)$, we get $\varphi \notin \operatorname{ad}_{\mathcal{T}}+\tilde{B}$. Since $\varphi(\mathcal{U})$ and $\tilde{B}(\mathcal{U})$ are contained in $[\mathcal{U}, \mathcal{U}]$, this implies by definition of $\mathcal{T}$ that we even have

$$
\varphi \notin \operatorname{ad}_{\mathcal{N}}+\tilde{B}
$$

as desired. (End of Case 2.)

Thus we have actually shown Theorem 2.2 for the case that $\Phi$ is not $p$-fold $\Gamma$ almost inner and that Conjecture 2.3 holds in dimension $p$. By Proposition 2.4 below, Conjecture 2.3 is true at least in dimension $p \leq 4$. This finishes the proof of Theorem 2.2.

2.3 Conjecture. Let $\mathcal{U}=\mathbf{Q}^{p}$. Denote the standard basis of $\mathcal{U}$ by $\left\{e_{1}, \ldots, e_{p}\right\}$ and its dual basis by $\left\{e_{1}^{*}, \ldots, e_{p}^{*}\right\} \subset \mathcal{U}^{*}$. Consider the canonical action of $G:=G l_{p}(\mathbf{Q})$ on $H:=\mathcal{U}^{*} \otimes \mathcal{U} \wedge \mathcal{U}$, given by $g(\lambda \otimes X \wedge Y)=\left({ }^{t} g^{-1}\right)(\lambda) \otimes g(X) \wedge g(Y)$. Let $\iota: H \rightarrow \mathcal{U}$ be the canonical contraction defined by $\iota(\lambda \otimes X \wedge Y)=\lambda(X) Y-\lambda(Y) X$. Let $f \in \operatorname{ker} \iota$. Then there exists $g \in G$ such that $g(f) \in S$, where

$$
S=\left\{f \in \operatorname{ker} \iota \mid\left\langle f, e_{i}^{*} \otimes e_{i} \wedge e_{j}\right\rangle=\left\langle f, e_{j}^{*} \otimes e_{i} \wedge e_{j}\right\rangle \text { for all } i, j \in\{1, \ldots, p\}\right\} .
$$

Here, $\langle.,$.$\rangle denotes the canonical scalar product on H$ associated with the standard scalar product on $\mathcal{U}$.

2.4 Proposition. Conjecture 2.3 is true for $p \leq 4$.

2.5 Remarks. (i) We will prove Proposition 2.4 only for $p \leq 3$ here (see (ii) below) because our proof for $p=4$ is quite tedious, although completely elementary. It turns out that in three of the six equations which characterize $S$ in case $p=4$, one can actually achieve to have zero on both sides. More explicitly: For $f \in \operatorname{ker} \iota$ there exists $g \in G$ such that

$$
\begin{gathered}
g(f) \perp e_{1}^{*} \otimes e_{1} \wedge e_{j}, e_{j}^{*} \otimes e_{1} \wedge e_{j} \quad \text { for } j=2,3,4, \text { and } \\
\left\langle g(f), e_{i}^{*} \otimes e_{i} \wedge e_{j}\right\rangle=\left\langle g(f), e_{j}^{*} \otimes e_{i} \wedge e_{j}\right\rangle \quad \text { for } i, j \in\{2,3,4\} .
\end{gathered}
$$

The strategy consists of two steps: First, find $\tilde{e}_{1} \in \mathcal{U}$ and a complementary subspace $\mathcal{U}^{\prime}$ of $\operatorname{span}\left\{\tilde{e}_{1}\right\}$ in $\mathcal{U}$ such that $f\left(\tilde{e}_{1}\right) \in \mathcal{U}^{\prime} \wedge \mathcal{U}^{\prime}$, where $f$ is viewed as an element of $\operatorname{Hom}(\mathcal{U}, \mathcal{U} \wedge \mathcal{U})$. In the second step, construct a basis $\left\{\tilde{e}_{2}, \tilde{e}_{3}, \tilde{e}_{4}\right\}$ of $\mathcal{U}^{\prime}$ such that, for the scalar product $\tilde{\zeta}_{.}, . \tilde{\rangle}$ with orthonormal basis $\left\{\tilde{e}_{1}, \ldots, \tilde{e}_{4}\right\}$, we get

$$
\begin{gathered}
f \tilde{\perp} \tilde{e}_{j}^{*} \otimes \tilde{e}_{1} \wedge \tilde{e}_{j} \quad \text { for } j=2,3,4, \text { and } \\
\left.\tilde{\langle} f, \tilde{e}_{i}^{*} \otimes \tilde{e}_{i} \wedge \tilde{e}_{j} \overline{\rangle}=\tilde{\langle} f, \tilde{e}_{j}^{*} \otimes \tilde{e}_{i} \wedge \tilde{e}_{j}\right\rangle \text { for } i, j \in\{2,3,4\} .
\end{gathered}
$$

(Then the map $g$ which sends $e_{1}, \ldots, e_{4}$ to $\tilde{e}_{1}, \ldots, \tilde{e}_{4}$ solves the problem.) This second step turns out to be equivalent to the following task: Let $a, b \in M_{3,3}(\mathbf{Q})$ 
such that $\operatorname{tr} a=0$ and $b$ is symmetric. Find $g \in G l_{3}(\mathbf{Q})$ such that the following two conditions are simultaneously satisfied: $g^{-1} a g$ has zero diagonal, and all offdiagonal entries of ${ }^{t} g b g$ are equal.

Both of the two steps just described can be solved by elementary methods, but involve some case distinctions.

(ii) For $p \leq 3$, Conjecture 2.3 is nearly trivial: In case $p=2$ we have $\operatorname{ker} \iota=0$, so there is nothing to show. In case $p=3$, consider the canonical identification of $H=\mathcal{U}^{*} \otimes \mathcal{U} \wedge \mathcal{U}$ with $\mathcal{U}^{*} \otimes \mathcal{U}^{*}$. Under this identification, ker $\iota$ corresponds to the space of symmetric bilinear forms on $\mathbf{Q}^{3}$, and $S$ corresponds to those symmetric bilinear forms $f$ for which $f\left(e_{1}, e_{2}\right)=f\left(e_{1}, e_{3}\right)=f\left(e_{2}, e_{3}\right)$. It is obvious that the $G_{3}(\mathbf{Q})$-orbit of a symmetric form $f$ indeed meets $S$ : For example, choose a basis of $\mathbf{Q}^{3}$ with respect to which $f$ has diagonal form (i.e., perform rational main axis transformation).

(iii) We do not know if Conjecture 2.3 is true for $p \geq 5$. Note that $H$, viewed as a representation space of $G$, splits into two irreducible components. One of these is ker $\iota$; the other one is isomorphic to the standard representation $\mathcal{U}=\operatorname{Im} \iota$. More specifically, ker $\iota$, viewed as a representation of $S l_{p}(\mathbf{Q})$, is the irreducible representation with highest weight $2 L_{1}+L_{2}+\cdots+L_{p-2}$ and highest weight vector $e_{1}^{*} \otimes e_{p-1} \wedge e_{p}$. Our conjecture says that every $G$-orbit (equivalently, every $S l_{p}(\mathbf{Q})$ orbit) in ker $\iota$ meets the subspace $S \subset H$. It seems to be a nontrivial problem to see whether this is true for $p \geq 5$.

(iv) If Conjecture 2.3 is true for a certain $p$, then in Theorem 2.2 we can replace the condition that $\Phi$ is not 4 -fold $\Gamma$-almost inner by the condition that $\Phi$ is not $p$-fold $\Gamma$-almost inner (and analogously in the Main Theorem). In particular, if Conjecture 2.3 should be true for every $p$, then we could completely drop that condition and only assume that $\Phi$ is not inner. Consequently, in the Main Theorem we could drop our additional assumption on $N$ and $\Gamma$. We do not claim, however, that Conjecture 2.3 is really necessary for this weakening of the assumptions. There might be other approaches to proving Theorem 2.2 which do not involve this conjecture.

\section{Proof of the Main Theorem}

In this section we will prove the Main Theorem 1.7, using the above Theorem 2.2 and Pesce's calculation of the spectrum of two-step nilmanifolds [Pe].

Let $N$ and $\Gamma$ be as in 1.7, and let $\left(\Gamma \backslash N, g_{t}\right)$ be a nontrivial isospectral deformation with left invariant metrics $g_{t}$. By 1.4 there exists a continuous family $\Phi_{t} \in \operatorname{AIA}(N ; \Gamma)$ such that $g_{t}=\Phi_{t}^{*} g_{0}$ and $\Phi_{0}=$ Id. Let $\mathcal{N}$ be the Lie algebra of $N$, and let $\varphi_{t}:=\log \Phi_{t * e}=\Phi_{t * e}-\operatorname{Id} \in \operatorname{AID}(\mathcal{N} ; \Gamma)$ (remember 2.1(vi)). Since $\left(\Gamma \backslash N, \Phi_{t}^{*} g\right)$ is a nontrivial deformation, there exists $t_{0}>0$ such that $\Phi_{t_{0}}$ is not inner and thus, by our additional assumption on $N$ and $\Gamma$, is not 4 -fold $\Gamma$-almost inner. By Theorem 2.2 there exists a horizontal 2 -form $\omega$ on $\mathcal{N}$ with $\omega(\log \Gamma, \log \Gamma) \subseteq 2 \mathbf{Z}$ such that no $\tilde{\Phi} \in \operatorname{Aut}\left(N_{\omega}\right)$ which extends $\Phi_{t_{0}}$ can be $\Gamma$-almost inner; equivalently, no $\tilde{\varphi} \in \operatorname{Der}\left(\mathcal{N}_{\omega}\right)$ which extends $\varphi_{t_{0}}$ can be $\Gamma$-almost inner. Note that for a given $\Gamma$-rational vector $X \in \mathcal{N}_{\omega}$, the condition $\tilde{\varphi}(X) \in\left[\mathcal{N}_{\omega}, X\right]_{\omega}$ is linear in $\tilde{\varphi}$. Thus there exists a finite set $\left\{X_{1}, \ldots, X_{r}\right\} \subset \mathcal{N}_{\omega}$ of $\Gamma_{\omega}$-rational vectors such that every derivation which is almost inner on each of these vectors must already be $\Gamma_{\omega}$-almost inner. In particular, every extension $\tilde{\varphi}$ of $\varphi_{t_{0}}$ must fail to be almost inner on at least one $X_{i}, i=1, \ldots, r$. (Note that in our proof of 2.2 we actually did construct a 
finite set with this property, namely, $\left\{X_{1}, \ldots, X_{p}, X_{1}+\cdots+X_{p}\right\}$.) We can assume

$$
W \notin\left[\mathcal{N}_{\omega}, X_{i}\right]_{\omega} \quad \text { for } i \in\{1, \ldots, r\}
$$

because $W \in\left[\mathcal{N}_{\omega}, X_{i}\right]_{\omega}$ would imply that every extension $\tilde{\varphi}$ of $\varphi_{t_{0}}$ is almost inner on $X_{i}$. (The $X_{i}$ from the proof of 2.2 satisfy (10) by construction.)

We have to construct a cocompact discrete subgroup $\tilde{\Gamma}$ of $N_{\omega}$ with $\pi(\tilde{\Gamma})=\Gamma$ such that the statement of Theorem 1.7 holds for the $S^{1}$-bundle $\pi: \tilde{\Gamma} \backslash N_{\omega} \rightarrow \Gamma \backslash N$. It will turn out that there is no reason for $\Gamma_{\omega}$ itself to already have this property.

Denote the center of $\mathcal{N}_{\omega}$ by $\mathcal{Z}_{\omega}$. Consider the above set $\left\{X_{1}, \ldots, X_{r}\right\}$ of $\Gamma_{\omega^{-}}$ rational vectors, and define linear subspaces $\mathcal{S}_{i}=\left[\mathcal{N}_{\omega}, X_{i}\right]_{\omega} \subseteq \mathcal{Z}_{\omega}$ and $\mathcal{S}_{i}^{W}=$ $\mathcal{S}_{i} \oplus \mathbf{R} W \subseteq \mathcal{Z}_{\omega}$. Note that $\mathcal{S}_{i}$ and $\mathcal{S}_{i}^{W}$ are $\Gamma_{\omega}$-rational subspaces of $\mathcal{N}_{\omega}$. Let $p_{i}: \mathcal{S}_{i}^{W} \rightarrow \mathbf{R} W$ denote projection along $\mathcal{S}_{i}$. Denote the inverse of $\exp _{\omega}: \mathcal{N}_{\omega} \rightarrow N_{\omega}$ by $\log _{\omega}$. For every $i \in\{1, \ldots, r\}$ define $k_{i} \in \mathbf{N}$ such that $p_{i}\left(\mathcal{S}_{i}^{W} \cap \log _{\omega} \Gamma_{\omega}\right) \subset \mathbf{R} W$ is generated by $\frac{1}{k_{i}} W$, and let $k \in \mathbf{N}$ be the least common multiple of the $k_{i}$. Define $W^{\prime}:=\frac{1}{k} W$ and $\tilde{\Gamma}:=\exp _{\omega}\left(\log \Gamma+\mathbf{Z} W^{\prime}\right) \supseteq \Gamma_{\omega}$. Obviously $\tilde{\Gamma}$ is again a cocompact discrete subgroup of $N_{\omega}$, and $\pi(\tilde{\Gamma})=\Gamma$. Define linear functionals $\mu_{i}: \mathcal{S}_{i}^{W} \rightarrow \mathbf{R}$ by

$$
\left.\mu_{i}\right|_{\mathcal{S}_{i}}=0, \quad \mu_{i}\left(W^{\prime}\right)=-1 .
$$

Note that our choice of $k$ guarantees that $\mu_{i}$ takes integer values on $\mathcal{S}_{i}^{W} \cap \log _{\omega} \tilde{\Gamma}$; we will need this later on. (This is the reason why we had to replace $\Gamma_{\omega}$ by the refined group $\tilde{\Gamma}$.) Now let $P:=\tilde{\Gamma} \backslash N_{\omega}$. We are going to show that the statement of Theorem 1.7 holds indeed for the $S^{1}$-bundle $\pi: P \rightarrow \Gamma \backslash N$.

Let $\mathcal{H}_{t}$ be any continuous family of left invariant connections in $P$. Denote by $\tilde{g}_{t}$ the left invariant metric associated with $g_{t}$ and $\mathcal{H}_{t}$ as in Remark 1.5; i.e., $\left.\tilde{g}_{t}\right|_{\mathcal{H}_{t}}=\pi^{*} g_{t}, \tilde{g}_{t}\left(\mathcal{H}_{t}, W^{\prime}\right)=0, \tilde{g}_{t}\left(W^{\prime}, W^{\prime}\right)=1$. These properties and the fact that $g_{t}=\left(\mathrm{Id}+\varphi_{t}\right)^{*} g_{0}$ imply that there exists a continuous family of linear maps $\tilde{\varphi}_{t}: \mathcal{N}_{\omega} \rightarrow \mathcal{N}_{\omega}$ such that $\pi \circ \tilde{\varphi}_{t}=\varphi_{t} \circ \pi, \tilde{\varphi}_{t}\left(W^{\prime}\right)=0$, and $\tilde{g}_{t}=\left(\mathrm{Id}+\tilde{\varphi}_{t}\right)^{*} \tilde{g}_{0}$. By equation (3) from $\S 1$, we only have to show that the spectrum of $\Delta_{\tilde{g}_{t}}$ on $C(P)^{S^{1}}$ is nonconstant in $t$.

Consider the quasi-regular unitary representation $\rho_{\tilde{\Gamma}}$ of $N_{\omega}$ on $L^{2}\left(\tilde{\Gamma} \backslash N_{\omega}, \mathbf{C}\right)$, defined by $\left(\rho_{\tilde{\Gamma}}(x) f\right)(y)=f(y x)$ for $x \in N_{\omega}, y \in \tilde{\Gamma} \backslash N_{\omega}, f \in L^{2}\left(\tilde{\Gamma} \backslash N_{\omega}, \mathbf{C}\right)$. Let $\lambda \in \mathcal{N}_{\omega}^{*}$ be a linear form which satisfies

$$
\begin{gathered}
\lambda\left(\mathcal{N}_{\omega}^{\lambda} \cap \log _{\omega} \tilde{\Gamma}\right) \subseteq \mathbf{Z}, \quad \text { where } \\
\mathcal{N}_{\omega}^{\lambda}:=\left\{X \in \mathcal{N}_{\omega} \mid\left[\mathcal{N}_{\omega}, X\right]_{\omega} \subseteq \operatorname{ker} \lambda\right\} .
\end{gathered}
$$

Then by $[\mathrm{Pe}]$ (Appendix A, Proposition 9), the irreducible unitary representation $\pi_{[\lambda]}$, associated in Kirillov theory with the co-adjoint orbit of $\lambda$, does occur in the decomposition of $\rho_{\tilde{\Gamma}}$. Let $V_{\lambda} \subseteq L^{2}\left(\tilde{\Gamma} \backslash N_{\omega}, \mathbf{C}\right)$ be an irreducible subspace such that $\left.\rho_{\tilde{\Gamma}}\right|_{V_{\lambda}} \sim \pi_{[\lambda]}$. It is well-known that $C(P)^{\lambda}:=V_{\lambda} \cap C^{\infty}(P, \mathbf{C})$ is dense in $V_{\lambda}$ and invariant under the Laplacian associated with any left invariant metric on $\tilde{\Gamma} \backslash N_{\omega}$. Moreover, $f\left(u \cdot \exp _{\omega} Z\right)=e^{2 \pi i \lambda(Z)} \cdot f(u)$ for all $f \in C(P)^{\lambda}, u \in P, Z \in \mathcal{Z}_{\omega}$. In particular, if $\lambda\left(W^{\prime}\right)=-1$, then by $(1)$

$$
C(P)^{\lambda} \subseteq C(P)^{S^{1}}
$$

Since the eigenvalues of $\Delta_{\tilde{g}_{t}}$ depend continuously on $t$, the theorem will be proved if we can show that there exists $\lambda \in \mathcal{N}_{\omega}^{*}$, satisfying (12), such that $\lambda\left(W^{\prime}\right)=-1$ and the spectrum of $\Delta_{\tilde{g}_{t}}$ on $C(P)^{\lambda}$ is nonconstant in $t$. 
Assume the contrary; i.e., no such $\lambda$ exists. Since $N_{\omega}$ is two-step nilpotent, we can use Pesce's calculation of the spectrum of compact Riemannian two-step nilmanifolds. The following actually contains a condensed repetition of part of Pesce's proof of the above Theorem 1.4 ([Pe], Propositions 6 and 7). By $[\mathrm{Pe}]$, Proposition 1, the spectrum of $\Delta_{\tilde{g}_{t}}$ on $C(P)^{\lambda}$ is equal to

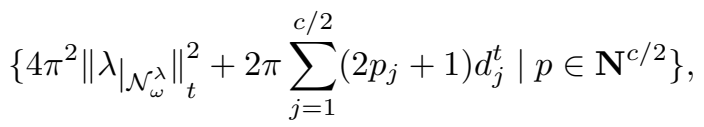

where $c$ is the codimension of $\mathcal{N}_{\omega}^{\lambda}$ in $\mathcal{N}_{\omega},\|\cdot\|_{t}$ is the norm on $\left(\mathcal{N}_{\omega}^{\lambda}\right)^{*}$ induced by $\left.\tilde{g}_{t}\right|_{\mathcal{N}_{\omega}^{\lambda}}$, and $\pm i d_{j}$ are the non-zero eigenvalues of $u_{t}^{\lambda}: \mathcal{N}_{\omega} \rightarrow \mathcal{N}_{\omega}$ which is defined by the equation $\tilde{g}_{t}\left(Y, u_{t}^{\lambda}(V)\right)=\lambda\left([Y, V]_{\omega}\right)$ for $Y, V \in \mathcal{N}_{\omega}$. Note that $\tilde{\varphi}_{t}\left(\mathcal{N}_{\omega}\right) \subseteq$ $\pi^{-1}\left(\varphi_{t}(\mathcal{N})\right) \subseteq \pi^{-1}([\mathcal{N}, \mathcal{N}]) \subseteq \mathcal{Z}_{\omega}$. Together with $\tilde{g}_{t}=\left(\mathrm{Id}+\tilde{\varphi}_{t}\right)^{*} \tilde{g}_{0}$, this implies that $u_{t}^{\lambda}$ is conjugate to $u_{0}^{\lambda}$ by $\left(\mathrm{Id}+\tilde{\varphi}_{t}\right)^{-1}$; in particular, the numbers $d_{j}^{t}$ are constant in $t$. Thus it follows from our assumption that

$$
\left\|\lambda_{\mathcal{N}_{\omega}^{\lambda}}\right\|_{t}^{2} \text { is constant in } t
$$

for every $\lambda$ satisfying (12) and $\lambda\left(W^{\prime}\right)=-1$.

Given such a $\lambda$, let $\left\{Y_{1}, \ldots, Y_{a}, Z_{1}, \ldots, Z_{b}, W^{\prime}\right\}$ be a $\tilde{g}_{0}$-orthonormal basis of $\mathcal{N}_{\omega}^{\lambda}$, where $\left\{Z_{1}, \ldots, Z_{b}, W^{\prime}\right\}$ is a $\tilde{g}_{0}$-orthonormal basis of $\mathcal{Z}_{\omega}$. Then

$$
\|\left.\lambda_{\left.\right|_{\mathcal{N}_{\omega}^{\lambda}} \|_{t}}\right|_{i=1} ^{a} \lambda\left(Y_{i}-\tilde{\varphi}_{t}\left(Y_{i}\right)\right)^{2}+\sum_{j=1}^{b} \lambda\left(Z_{j}-\tilde{\varphi}_{t}\left(Z_{j}\right)\right)^{2}+1
$$

Define $A_{\lambda}=\left(\lambda\left(Y_{1}\right), \ldots, \lambda\left(Z_{b}\right)\right) \in \mathbf{R}^{a+b}$ and $B_{\lambda}(t):=\left(\lambda\left(\tilde{\varphi}_{t}\left(Y_{1}\right)\right), \ldots,\left(\tilde{\varphi}_{t}\left(Z_{b}\right)\right)\right) \in$ $\mathbf{R}^{a+b}$. Since our assumption implies that the right hand side of (13) must be constant in $t$, we get

$$
B_{\lambda}(t) \perp-2 A_{\lambda}+B_{\lambda}(t) \text { for every } t,
$$

where $\perp$ denotes orthogonality with respect to the standard scalar product on $\mathbf{R}^{a+b}$.

Consider $\lambda \in \mathcal{N}_{\omega}^{*}$ such that $\lambda\left(\log _{\omega} \tilde{\Gamma}\right) \subseteq \mathbf{Z}$ (in particular, $\lambda$ satifies (12)) and $\lambda\left(W^{\prime}\right)=-1$. For every $\alpha \in \mathcal{N}_{\omega}^{*}$ such that $\alpha\left(\log _{\omega} \tilde{\Gamma}\right) \subseteq \mathbf{Z}$ and $\left.\alpha\right|_{\mathcal{Z}_{\omega}}=0$, the form $\lambda+\alpha$ again satisfies those two conditions. Note that $\mathcal{N}_{\omega}^{\lambda}=\mathcal{N}_{\omega}^{\lambda+\alpha}$. Moreover, $B_{\lambda+\alpha}(t)=B_{\lambda}(t)$, because $\tilde{\varphi}_{t}\left(\mathcal{N}_{\omega}\right) \subseteq \mathcal{Z}_{\omega}$. Since both $\lambda$ and $\lambda+\alpha$ must satisfy (14), by forming differences on the right hand side we get $B_{\lambda}(t) \perp A_{\alpha}$ for every $t$. We have $A_{\alpha}=\left(\alpha\left(Y_{1}\right), \ldots, \alpha\left(Y_{a}\right), 0, \ldots, 0\right)$ by choice of $\alpha$. But the vectors $\left(\alpha\left(Y_{1}\right), \ldots, \alpha\left(Y_{a}\right)\right)$, where $\alpha$ ranges over all linear forms with the above properties, span the whole $\mathbf{R}^{a}$. Thus $B_{\lambda}(t)$ has the form $\left(0, \ldots, 0, \lambda\left(\tilde{\varphi}_{t}\left(Z_{1}\right)\right), \ldots, \lambda\left(\tilde{\varphi}_{t}\left(Z_{b}\right)\right)\right)$. Let $A_{\lambda}^{\mathcal{Z}}=\left(\lambda\left(Z_{1}\right), \ldots, \lambda\left(Z_{b}\right)\right), B_{\lambda}^{\mathcal{Z}}(t)=\left(\lambda\left(\tilde{\varphi}_{t}\left(Z_{1}\right)\right), \ldots, \lambda\left(\tilde{\varphi}_{t}\left(Z_{b}\right)\right)\right)$. Now (14) implies

$$
B_{\lambda}^{\mathcal{Z}}(t) \perp-2 A_{\lambda}^{\mathcal{Z}}+B_{\lambda}^{\mathcal{Z}}(t) \quad \forall t
$$

for all $\lambda \in \mathcal{N}_{\omega}^{*}$ with $\lambda\left(\log _{\omega} \tilde{\Gamma}\right) \subseteq \mathbf{Z}$ and $\lambda\left(W^{\prime}\right)=-1$. For $\beta \in \mathcal{N}_{\omega}^{*}$ with $\beta\left(\log _{\omega} \tilde{\Gamma}\right) \subseteq \mathbf{Z}$ and $\beta\left(W^{\prime}\right)=0$, the form $\lambda+\beta$ still has these two properties of $\lambda$. Moreover, $B_{\lambda+\beta}^{\mathcal{Z}}(t)=B_{\lambda}^{\mathcal{Z}}(t)$, because $\tilde{\varphi}_{t}\left(\mathcal{Z}_{\omega}\right) \subseteq \pi^{-1}\left(\varphi_{t}(\mathcal{Z})\right)=\pi^{-1}\{0\}=\mathbf{R} W^{\prime}$. Forming differences on the right hand side of (15), we get $B_{\lambda}^{\mathcal{Z}}(t) \perp A_{\beta}^{\mathcal{Z}}$ for all such $\beta$. But the vectors $A_{\beta}^{\mathcal{Z}}$ span the whole $\mathbf{R}^{b}$; hence $B_{\lambda}^{\mathcal{Z}}(t)=0$ and thus $B_{\lambda}(t)=0$. Note that this implies that the $\tilde{\varphi}_{t}$ are actually derivations. Moreover, $B_{\lambda}(t)=0$ just means 


$$
\begin{gathered}
\lambda\left(\tilde{\varphi}_{t}(X)\right)=0 \text { for all } t \geq 0, X \in \mathcal{N}_{\omega}^{\lambda}, \lambda \in \mathcal{N}_{\omega}^{*} \\
\text { with } \lambda\left(\log _{\omega} \tilde{\Gamma}\right) \subseteq \mathbf{Z}, \lambda\left(W^{\prime}\right)=-1 .
\end{gathered}
$$

But for our above $t_{0}$, there exists $i_{0} \in\{1, \ldots, r\}$ such that $\tilde{\varphi}_{t_{0}}\left(X_{i_{0}}\right) \notin\left[\mathcal{N}_{\omega}, X_{i_{0}}\right]_{\omega}=$ $\mathcal{S}_{i_{0}}$. On the other hand, $\tilde{\varphi}_{t_{0}}\left(X_{i_{0}}\right) \in \mathcal{S}_{i_{0}}^{W}$, since $\tilde{\varphi}_{t_{0}}$ extends $\varphi_{t_{0}}$ which is $\Gamma$-almost inner. In particular, $\mu_{i_{0}}\left(\tilde{\varphi}_{t_{0}}\left(X_{i_{0}}\right)\right) \neq 0$ for the corresponding form $\mu_{i_{0}}$ defined in (11). Extend $\mu_{i_{0}}$ to a linear form $\lambda \in \mathcal{N}_{\omega}^{*}$ such that $\lambda\left(\log _{\omega} \tilde{\Gamma}\right) \subseteq \mathbf{Z}$; that is possible because $\mu_{i_{0}}$ already takes integer values on $\mathcal{S}_{i_{0}}^{W} \cap \log _{\omega} \tilde{\Gamma}$ by construction of $\tilde{\Gamma}$. Now $X_{i_{0}} \in \mathcal{N}_{\omega}^{\lambda}$ because $\left.\lambda\right|_{\mathcal{S}_{i_{0}}}=0$; moreover $\lambda\left(W^{\prime}\right)=-1$ and $\lambda\left(\tilde{\varphi}_{t_{0}}\left(X_{i_{0}}\right)\right) \neq 0$. This is a contradiction to (16). Hence our assumption that $\Delta_{\tilde{g}_{t}}$ has constant spectrum on each $C(P)^{\lambda}$ with $\lambda\left(\log _{\omega} \tilde{\Gamma}\right) \subseteq \mathbf{Z}$ and $\lambda\left(W^{\prime}\right)=-1$ must have been false. This finishes the proof.

\section{REFERENCES}

[DG1] D. DeTurck, C. Gordon, Isospectral deformations I: Riemannian structures on twostep nilspaces, Comm. on Pure and Appl. Math. XL (1987), 367-387. MR 88m:58186

[DG2] D. DeTurck, C. Gordon, Isospectral deformations II: trace formulas, metrics, and potentials, Comm. on Pure and Appl. Math. 42 (1989), 1067-1095. MR 91e:58197

[DGGW1] D. DeTurck, H. Gluck, C. Gordon, D. Webb, The inaudible geometry of nilmanifolds, Invent. Math. 111 no. 2 (1993), 271-284. MR 93k:58222

[DGGW2] D. DeTurck, H. Gluck, C. Gordon, D. Webb, The geometry of isospectral deformations, Proc. of Symposia in Pure Mathematics 54 no. 3 (1993), 135-154. MR 94b:58096

[Eb] P. Eberlein, Geometry of two-step nilpotent groups with a left invariant metric, Ann. Sci. de l'Ecole Norm. Sup., 4ème série, t. 27 (1994), 611-660. MR 95m:53059

[Gi] P. Gilkey, On spherical space forms with meta-cyclic fundamental group which are isospectral but not equivariant cobordant, Compositio Mathematica 56 (1985), 171200. MR 87a:58158

[Go] C. Gordon, The Laplace spectra versus the length spectra of Riemannian manifolds, Contemp. Math 51 (1986), 63-80. MR 87i:58170

[GOS] C. Gordon, H. Ouyang, D. Schueth, Distinguishing isospectral manifolds by bundle Laplacians, Math. Res. Lett. 4 (1997), 23-33. CMP 97:08

[GW] C. Gordon, E. Wilson, Isospectral deformations of compact solvmanifolds, J. Diff. Geom. 19 (1984), 241-256. MR 85j:58143

[Ik] A. Ikeda, On lens spaces which are isospectral but not isometric, Ann. Scient. Ec. Norm. Sup. 13 (1980), 303-315. MR 83a:58091

$[\mathrm{KN}] \quad$ S. Kobayashi, K. Nomizu, Foundations of differential geometry, Vol. 1, Interscience, New York, 1963. MR 27:2945

[No] K. Nomizu, On the cohomology of compact homogeneous spaces of nilpotent LieGroups, Ann. of Math. 59 (1954), 531-538. MR 16:219c

[OP] H. Ouyang, H. Pesce, Déformations isospectrales sur les nilvariétés de rang deux, C. R. Acad. Sci. Paris, sér. I Math. 314 (1992), 621-623. MR 93g:58152

$[\mathrm{Ou}] \quad \mathrm{H}$. Ouyang, On isospectral deformations on two-step nilmanifolds, Ph.D. thesis, Washington University, St. Louis, 1991.

[Pe] H. Pesce, Calcul du spectre d'une nilvariété de rang deux et applications, Trans. AMS 339 (1993), 433-461. MR 93k:58227

[Ra] M.S. Raghunathan, Discrete Subgroups of Lie groups, Springer, Berlin/New York, 1972. MR 58:22394a

[Sch] D. Schueth, Isospectral deformations on Riemannian manifolds which are diffeomorphic to compact Heisenberg manifolds, Comment. Math. Helvetici 70 (1995), 434-454. MR 96g:58200

[We] R.O. Wells, Differential analysis on complex manifolds, Springer, New York, 1980. MR 83f: 58001

Mathematisches Institut der Univ. Bonn, Beringstr. 1, D-53115 Bonn, Germany

E-mail address: schueth@math.uni-bonn.de 